Original Research Paper

\title{
Tracking Algorithm for Estimating the Orientation Angles of the Object Based on the Signals of Satellite Radio Navigation System
}

\author{
Alexandr Perov \\ Moscow Power Engineering Institute, National Research University, Moscow, Russian Federation
}

Article history

Received: 19-01-2015

Revised: 02-12-2012

Accepted: 03-12-2015

Email: russian-science@mail.ru

\begin{abstract}
In the article, the theory of optimal filtering of information processes is used to synthesize optimal one-stage estimation algorithm for the object orientation angles using the signals of the satellite navigation system. The article also provides equations of optimal filtering, as well as their representation in the form of the tracking system comprising of an orientation angles discriminator and a smoothing filter. The synthesized algorithm has no tracking systems for the phases of the received signals (or phase differences) and the procedures for resolving the ambiguity of phase measurements that enhance its noise immunity while maintaining high precision in estimating the orientation angles. The article provides expressions for the calculation of the Cramer-Rao lower limit for the dispersion errors matrix for estimating object orientation angles when receiving signals of an arbitrary number of navigation satellites. The results of the simulation of the synthesized system are provided in the study.
\end{abstract}

Keywords: Satellite Navigation, Angular Orientation, Tracking, Optimal Algorithm, Synthesis, Potential Accuracy

\section{Introduction}

Satellite navigation systems are now widely used for a variety of technical problems, including extracting information from the phase of the received signals (Misra and Enge, 2012; Kaplan and Hegarty, 2006). One of such application is the determination of the orientation angles of the object on the signals of satellite radio navigation system, taken spaced points (Cohen, 1992; Parkinson and Spilker, 1996). The approach is because the signals coming from the navigation satellite in two spaced points have a phase shift depending on the angle between the reference line connecting the two points of reception and the direction of arrival of the satellite navigation signal. Measuring this angle and knowing the direction of satellite navigation, we can determine the orientation angle of the reference line. To implement this approach, navigation receivers are placed in the receiving points, which measure the phases of the received navigation signals. Further, the phase difference is formed, which carries information about the angle between the reference line and the direction of arrival of the signal from the navigation satellite. The main problem consists in that the measured phase difference is different from the true phase difference proportional to the phase difference between the arrival times of the signal phase edge in the receiving point on an integer number of periods of the high-frequency filling. That is, there is ambiguity of phase measurements. Various algorithms for ambiguity resolution of phase measurements are presented extensively in literature (Povalyaev, 2008; Lipkin, 2008; Boriskin et al., 2010; Perov and Kharisov, 2010; Knight, 1994; Hodgart and Purivigraipong, 2000; Lin et al., 2004; Verhagen and Teunissen, 2006).

The described ideology for determining the orientation angles using signals of satellite navigation systems is based on the principle of two-stage processing of signals from navigation satellites (Perov and Kharisov, 2010), in which the first stage is estimation of parameters of the received radio signals (delay, phase and the Doppler frequency shift) and the second stage solves the navigation task-formation of estimations of coordinates, velocity and orientation angles of the consumer. At the same time in relation to a standard navigation equipment, recently there are proposed and studied one-stage processing algorithms, where, in a single complex filter processing the signals of all visible navigation satellites, the desired estimations of position and velocity of the consumer (Perov, 2004) are formed. One-stage algorithm has no intermediate stage of 
forming pseudo-range and pseudo phases estimations and estimations of the desired parameters are formed by processing the outputs of discriminators (temporal and phase), which allows disambiguating phase measurements for prolonged observation time. This ideology, in principle, can be used to solve the problem of estimating the orientation angles of the object at the reception of navigation signals on several spatially separated antennas. The paper by (Perov and Kharisov, 2010) developed this approach for the task of determining the orientation of the reference line in a vertical plane and shown to be effective. This article contains the synthesis and analysis of the one-stage tracking algorithm for estimating the orientation angles of the object in space with the signal processing of all visible navigation satellites.

\section{Research Methodology}

\section{Statement of the Problem for Synthesis}

Let us consider a geocentric coordinate system $O X Y Z$ rigidly connected with the Earth (1) and the triangle $A B C$ defining the reference plane, which bind tightly Coordinate System (CS) $O_{c} X_{c} Y_{c} Z_{c}$, the centre of which $O_{c}$ lies in the plane $A B C$, axis $O_{c} X_{c}$ is directed along the line $A B$, axis $O_{c} \mathrm{Y}_{\mathrm{c}}$ lies in the reference plane, axis $O_{c} \mathrm{Z}_{\mathrm{c}}$ completes the system of coordinates to the right hand.

Orientation of a triangle $A B C$ in CS $O X Y Z$ is given by the Euler angles $\alpha_{1}, \alpha_{2}$ and $\alpha_{3}$ (roll, pitch and yaw, respectively) or in the vector form $\alpha=\left|\alpha_{1} \alpha_{2} \alpha_{3}\right|^{T}$, which are the rotation angles in $\mathrm{CS} O_{c} X_{c} Y_{c} Z_{c}$, relatively to $\mathrm{CS} O X Y Z$.

Assume that the triangle $A B C$ moves in CS $O X Y Z$, so that the orientation angles vector varies in time, i.e., $\alpha(t)$.

Points $A, B$ and $C$ are used to receive radio signals from $n$ navigation satellites.

The result of processing the received signals is the task to synthesize the optimal filtration system for vector angles $\alpha(t)$.

Let us introduce the directing cosines of the $i$ th navigation satellite, identifying them, for example, relatively to the point $O_{c}$ and assuming they are the same for all the other points of the triangle $A B C$ (which is permissible for small triangles relatively to distances to the navigation satellites):

$$
\mu_{x i}=\frac{x_{i}-x_{O_{\mathrm{c}}}}{R_{i}}, \mu_{y i}=\frac{y_{i}-y_{O_{\mathrm{c}}}}{R_{i}}, \mu_{z i}=\frac{z_{i}-z_{O_{\mathrm{c}}}}{R_{i}}
$$

where, $x_{O_{\mathrm{c}}}, y_{O_{\mathrm{c}}}, z_{O_{\mathrm{c}}}$ are coordinates of the point $O_{c}$ in CS $O X Y Z ; R_{i}$ is the distance to $i$-th navigation satellite. Let us introduce unit vectors $\mu_{H C}, i=\left|\mu_{x i}, \mu_{y i}, \mu_{z i}\right|^{T}$, $i=\overline{1, n}$ to $\mathrm{CS} O X Y Z$ and unit vectors $l_{A O_{c}, c}, l_{B O_{c}, c}, l_{C O_{c}, c}$, specifying the direction of points $A, B$ and $C$ respectively to the point $O_{c}$ in $\operatorname{CS} O_{c} X_{c} Y_{c} Z_{c}$.
Let us transform unit vectors $l_{A O_{c}, c}, l_{B O_{c}, c}, l_{C O_{c}, c}$, set in CS $O_{c} X_{c} Y_{c} Z_{c}$ into vectors $1_{\mathrm{AO}_{\mathrm{c}}, 3}, l_{B O_{c}, 3}, l_{C O_{c}, 3}$ in CS OXYZ:

$$
\begin{aligned}
& l_{A O_{c}, 3}(\alpha)=U_{c}^{3}(\alpha) l_{A O_{c}, c}, \\
& l_{B O_{c}, 3}(\alpha)=U_{c}^{3}(\alpha) l_{B O_{c}, c}, l_{C O_{c}, 3}(\alpha)=U_{c}^{3}(\alpha) l_{C O_{c}, c}
\end{aligned}
$$

where, $U_{c}^{3}$ is transformation matrix for coordinates from $\mathrm{CS} O_{c} X_{c} Y_{c} Z_{c}$ to CS $O X Y Z$, defined as:

$$
\begin{aligned}
& U_{\mathrm{c}}^{3}(\alpha)=\left|\begin{array}{ccc}
\cos \left(\alpha_{3}\right) & -\sin \left(\alpha_{3}\right) & 0 \\
\sin \left(\alpha_{3}\right) & \cos \left(\alpha_{3}\right) & 0 \\
0 & 0 & 1
\end{array}\right| . \\
& \left|\begin{array}{ccc}
\cos \left(\alpha_{2}\right) & 0 & \sin \left(\alpha_{2}\right) \\
0 & 1 & 0 \\
-\sin \left(\alpha_{2}\right) & 0 & \cos \left(\alpha_{2}\right)
\end{array}\right| \cdot\left|\begin{array}{ccc}
1 & 0 & 0 \\
0 & \cos \left(\alpha_{1}\right) & -\sin \left(\alpha_{1}\right) \\
0 & \sin \left(\alpha_{1}\right) & \cos \left(\alpha_{1}\right)
\end{array}\right|
\end{aligned}
$$

Let us consider satellite radio signal with code division coming from $i$-th navigation satellite to the point $O_{c}$ for which let us write the expression:

$$
s_{0, i}(t)=A h_{\not \kappa, i}\left(t-\tau_{0, i}\right) \cos \left(\left(\omega_{0}+\omega_{x, i}\right) t+\pi \vartheta_{\mathrm{HC}, i}+\varphi_{0, i}\right)
$$

where, $A$ is the amplitude; $\omega_{i}$ is the signal carrying

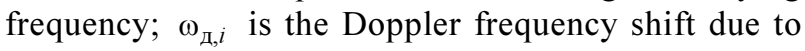
the movement of the point $A\left(O_{c}\right)$ of the triangle $A B C$; $\tau_{0, i}$ is the delay of the envelope curve of the signal at its distribution from the navigation satellite to receiver, $\varphi_{0, \mathrm{i}}$ is the initial phase of the signal received from the $i$-th navigation satellite, $\omega_{\text {д, } i}$ is the Doppler signal frequency shift; $h_{\text {дк, }}(t)$ is the function of modulation by the ranging code, $\vartheta_{H C, i}$ are the navigation data taking the value of 0 or 1 .

Representation (2) is valid for a certain time interval $T$ in which the parameters $\omega_{д, i}, \tau_{0, i}$ can be considered as constant.

At points $A, B$ and $C$, the received signals are shifted in phase relatively to similar signals received at the point $O_{c}$ into the phase angles:

$$
\begin{aligned}
\psi_{\mathrm{A}, \mathrm{i}}(\alpha) & =\frac{2 \pi \mathrm{L}_{\mathrm{AO}_{c}} \mu_{H C, \mathrm{i}}^{m} l_{\mathrm{AO}_{c}, 3}(\alpha)}{\lambda} \\
\psi_{\mathrm{B}, \mathrm{i}}(\alpha) & =\frac{2 \pi \mathrm{L}_{\mathrm{BO}_{c}} \mu_{H C, \mathrm{i}}^{m} l_{\mathrm{BO}_{c}, 3}(\alpha)}{\lambda}, \psi_{\mathrm{c}, \mathrm{i}}(\alpha)=\frac{2 \pi \mathrm{L}_{\mathrm{CO}_{c}} \mu_{H C, \mathrm{i}}^{m} l_{\mathrm{CO}_{c}, 3}(\alpha)}{\lambda}
\end{aligned}
$$

where $L_{A O_{c}}, L_{B O_{c}}, L_{C O_{\mathrm{c}}}$ mean the distance between the exact $O_{c}$ and points $A, B$ and $C$ respectively.

Therefore, it is possible to write (for the same time slot $T$ ) the following: 


$$
\begin{aligned}
& s_{A, i}(t)=A h_{\mathbb{\alpha}, i}\left(t-\tau_{A, i}\right) \\
& \cos \left(\left(\omega_{0}+\omega_{\nexists, i}\right) t+\pi \vartheta_{\mathrm{HC}, i}+\varphi_{0, i}+\psi_{A, i}(\alpha)+\omega_{A, \psi, i} t\right), \\
& s_{B, i}(t)=A h_{\mathbb{\alpha}, i}\left(t-\tau_{B, i}\right) \\
& \cos \left(\left(\omega_{0}+\omega_{\alpha, i}\right) t+\pi \vartheta_{\mathrm{HC}, i}+\varphi_{0, i}+\psi_{B, i}(\alpha)+\omega_{B, \psi, i} t\right), \\
& s_{C, i}(t)=A h_{\nexists \kappa, i}\left(t-\tau_{C, i}\right) \\
& \cos \left(\left(\omega_{0}+\omega_{\nexists, i}\right) t+\pi \vartheta_{\mathrm{HC}, i}+\varphi_{0, i}+\psi_{C, i}(\alpha)+\omega_{C, \psi, i} t\right)
\end{aligned}
$$

where, $\omega_{A, \psi, i}, \omega_{B, \psi, i}, \omega_{C, \psi, i}$ are the Doppler signals frequency shifts in points $A, B$ and $C$ respectively due to the rotation of the triangle $A B C$ relatively the point $O_{c}$, $\tau_{A, i}, \tau_{B, i}, \tau_{C, i}$ is the delays of the envelope curve of the signal from the $i$-th NS in the receiving points $A, B$ and $C$.

Strictly speaking, the delays $\tau_{A, i}, \tau_{B, i}, \tau_{C, i}$ depend on the vector of angles $\alpha$. However, the consideration of this dependence has little effect on the accuracy of estimating the orientation angles of the phase of the carrier frequency $\left(\psi_{A, i}(\alpha),\left(\psi_{B, i}(\alpha),\left(\psi_{C, i}(\alpha)\right)\right.\right.$. Thus, this dependence is not taken into account further.

When receiving $n$ satellite radio signals, the total signal over small time intervals (for example, equal to the period ranging code) are described by the following expressions:

$$
\begin{aligned}
& s_{A}(t)=\sum_{i=1}^{n} s_{A, i}(t) \\
& =\sum_{i=1}^{n} A h_{\text {aא,i }}\left(t-\tau_{A, i}\right) \cos \left(\begin{array}{l}
\left(\omega_{0}+\omega_{\pi, i}\right) \\
t+\pi \vartheta_{\mathrm{HC}, i}+\varphi_{0, i}+\psi_{A, i}(\alpha)+\omega_{A, \psi, i} t
\end{array}\right), \\
& s_{B}(t)=\sum_{i=1}^{n} s_{B, i}(t) \\
& =\sum_{i=1}^{n} A h_{\mathbb{A}, i}\left(t-\tau_{B, i}\right) \cos \left(\begin{array}{l}
\left(\omega_{0}+\omega_{\nexists, i}\right) \\
t+\pi \vartheta_{\mathrm{HC}, i}+\varphi_{0, i}+\psi_{B, i}(\alpha)+\omega_{B, \psi, i} t
\end{array}\right), \\
& s_{C}(t)=\sum_{i=1}^{n} s_{C, i}(t) \\
& =\sum_{i=1}^{n} A h_{\mathrm{ak}, i}\left(t-\tau_{C, i}\right) \cos \left(\begin{array}{l}
\left(\omega_{0}+\omega_{a, i}\right) \\
t+\pi \vartheta_{\mathrm{HC}, i}+\varphi_{0, i}+\psi_{C, i}(\alpha)+\omega_{C, \psi, i} t
\end{array}\right)
\end{aligned}
$$

Let us write the equations of observation in the points $A, B$ and $C$ :

$$
\begin{aligned}
& y_{A}(t)=s_{A}(t)+n_{A}(t), y_{B}(t) \\
& =s_{B}(t)+n_{B}(t), y_{C}(t)=s_{C}(t)+n_{C}(t)
\end{aligned}
$$

where, $n_{A}(t), n_{B}(t)$ and $n_{C}(t)$ are independent white Gaussian noises with equal bilateral spectral densities $N_{0} / 2$.

Let us introduce the vector surveillance $\mathrm{y}(\mathrm{t})=y_{A}(t)$ $\left.y_{B}(t) y_{C}(t)\right|^{T}$, for which let us write:

$$
\mathrm{y}(t)=\mathrm{s}(t)+\mathrm{n}(t)
$$

where, $\mathrm{s}(\mathrm{t})=\left|s_{A}(t) s_{B}(t) s_{C}(t)\right|^{T}, n(t)=\left|s_{A}(t) s_{B}(t) s_{C}(t)\right|^{T}$ is white Gaussian noise vector with a matrix of bilateral spectral densities $N_{0}=I N_{0} / 2$.

Let us assume that receivers carry synchronous sampling of the input process in time, so that the processing system receives an implementation in discrete time $t_{k, i}$ (Fig. 2):

$$
\mathrm{y}_{k, i}=s\left(t_{k, i}, \psi_{k, i}\right)+n_{k, i}
$$

where, $t_{k, i}=k T+i T_{d} ; T=N T_{d}$ is a step of discrete processing in the tracking system loop; $T_{d}$ is a discretisation period in an analogue-digital converter; $n_{k, i}$ is a vector of independent discrete white Gaussian noises with equal dispersions $\sigma_{n}^{2}=\frac{N_{0}}{2 T_{d}}$, where $N_{0}$ is one-sided power spectral density of the internal noise of the receiver.

The dynamics of change of the orientation angles is defined by equations in sampling times $t_{k}$ :

$$
\alpha_{k}=\alpha_{k-1}+T \mathrm{v}_{\alpha, k-1}, \mathbf{v}_{\alpha, k}=\mathbf{v}_{\alpha, k-1}+\xi_{\alpha, k-1}
$$

where, $\xi \alpha, k-1$ is the vector of discrete white Gaussian noise with dispersion matrix $D_{\xi}$.

\section{Synthesis of Optimal Filtering Algorithm for Orientation Angles}

Let us introduce a state vector $\mathrm{X}_{k}=\left|\begin{array}{c}\alpha_{k} \\ \mathrm{v}_{\alpha, k}\end{array}\right|$, for which let us write the matrix equation:

$$
X_{k}=F X_{k-1}+G \xi_{\alpha, k-1}
$$

where, $\mathrm{F}=\left|\begin{array}{cc}\mathrm{I} & \mathrm{TI} \\ 0 & \mathrm{I}\end{array}\right|, \mathrm{G}=\left|\begin{array}{l}0 \\ \mathrm{I}\end{array}\right|, I$ is an identity matrix with a size of $3 \times 3$.

In this problem, the observations $\mathrm{Y}_{k-1,1}^{k}=\left\{\mathrm{y}_{k-1,1,} \mathrm{y}_{k-1,2}, \ldots, \mathrm{y}_{k-1, N}\right\}$ the time interval $\left[t_{k-1}, 1, t_{k}\right]$ (Fig. 1) give information about the state vector $X_{k-1}$, relevant to the time $t_{k-1}$. Therefore, if we are interested in assessing the current state vector $x_{k-1}$ according to the observations $Y_{0}^{k}=\left\{Y_{0,1}^{1}, Y_{1,1}^{2}, \ldots, Y_{k-1,1}^{k}\right\}$, it is necessary to consider the a posteriori probability density $p\left(\mathrm{X}_{k-1} \mid \mathrm{Y}_{0}^{k}\right)$, for which it is possible to write the equations (Tikhonov and Kharisov, 2004; Perov, 2012):

$$
\begin{aligned}
& p\left(X_{k-1} \mid Y_{0}^{k}\right)=c p\left(X_{k-1} \mid Y_{0}^{k-1}\right) p\left(Y_{k-1,1}^{k} \mid X_{k-1}\right), \\
& p\left(X_{k-1} \mid Y_{0}^{k-1}\right)=\int_{-\infty}^{\infty} p\left(X_{k-2} \mid Y_{0}^{k-1}\right) p\left(X_{k-1} \mid X_{k-2}\right) d X_{k-2}
\end{aligned}
$$




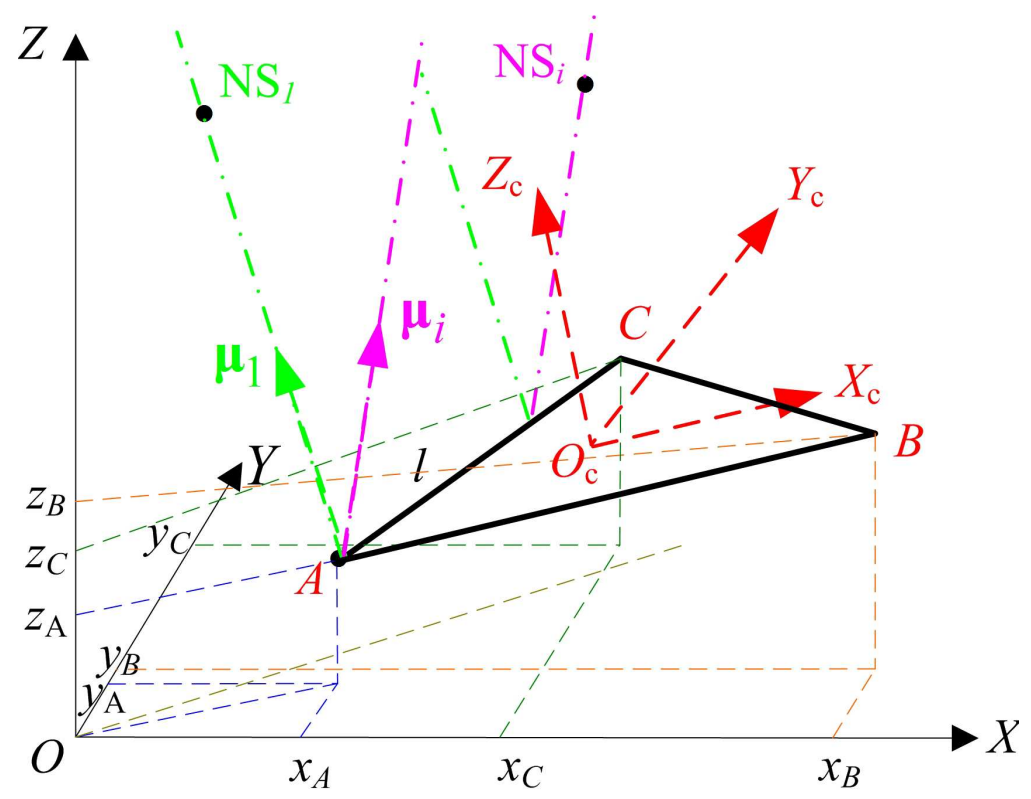

Fig. 1. Geometric scheme of the objectives

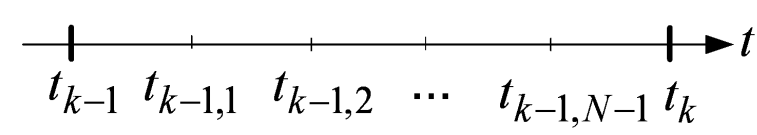

Fig. 2. Time indexing scheme

Therefore, at the time $t_{k}$, processing all of the available to the time observations $Y_{0}^{k}$, we will form a state vector estimate $\hat{X}_{k-1}$, corresponding to the state vector $X_{k-1}$ at the time $t_{k-1}$

Let us write the equations of optimal filtering of the vector $X_{k}$ in the Gaussian approximation (Tikhonov and Kharisov, 2004), assuming the delays $\tau_{A, i}, \tau_{B, i}, \tau_{C, i}$ and Doppler frequency shifts $\omega_{x, i}, \omega_{B, \psi, i}, \quad \omega_{C, \psi, i}, \quad i=\overline{1, n}$ known as:

$\hat{X}_{k}=\tilde{X}_{k}+D_{X, k}\left(\frac{\partial F_{k}\left(\tilde{X}_{k}\right)}{\partial X}\right)^{\mathrm{T}}$

$\tilde{X}_{k}=F \hat{X}_{k-1}$,

$\tilde{D}_{X, k}=F D_{X, k-1} F^{\mathrm{T}}+G D_{\xi} G^{\mathrm{T}}, D_{X, k}^{-1}=\tilde{D}_{x, k}^{-1}-\frac{\partial}{\partial X}\left(\frac{\partial F_{k}\left(\tilde{X}_{k}\right)}{\partial X}\right)^{\mathrm{T}}$

Where:

$\hat{X}_{k}=$ The estimation of the filtered process

$\hat{X}_{k}=$ The extrapolated process estimation

$D_{X, k}=$ The filtering errors dispersion matrix

$\tilde{D}_{X, k}=$ The extrapolation errors dispersion matrix:

$$
F_{k}\left(X_{k}\right)=\ln p\left(Y_{k, 1}^{k+1} \mid X_{k}\right)
$$

In (5), (6) the derivative of the scalar according to vector is understood as a row vector (Perov and Kharisov, 2010).

Let us write signal functions (4) in the discrete time for the moments $t_{k, j} \in\left[t_{k, 1}, t_{k, M}\right]$, where $t_{k, j}=t_{k}+j T_{d}, T_{d}$ is the discretisation step for analogue-to-digital converter, $M T_{d}=T$ is the time interval, multiple to periods of the ranging code:

$$
\begin{aligned}
& S_{A ; k, l}=\sum_{i=1}^{n} A h_{д \kappa, i}\left(t_{k, l}-\tau_{A, i ; k}\right) \cos \left(\begin{array}{l}
\left(\omega_{0}+\omega_{\lambda, i, k}\right) t_{k, l}+\pi \vartheta_{\mathrm{HC}, i ; k} \\
+\varphi_{0, i}+\psi_{A, i}\left(\alpha_{k}\right)+\omega_{A, \psi, i, k} t_{k, l}
\end{array}\right) \text {, }
\end{aligned}
$$

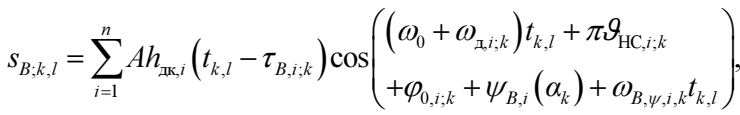

$$
\begin{aligned}
& s_{C ; k, l}=\sum_{i=1}^{n} A h_{\text {वk,i }}\left(t_{k, l}-\tau_{C, i, k}\right) \cos \left(\begin{array}{l}
\left(\omega_{0}+\omega_{\text {да, } i, k}\right) t_{k, l}+\pi \vartheta_{\mathrm{HC}, i ; k} \\
+\varphi_{0, i}+\psi_{C, i}\left(\alpha_{k}\right)+\omega_{C, \psi, i, k} t_{k, l}
\end{array}\right)
\end{aligned}
$$

In these equations, parameters $\varphi_{0, i, k}$ and $\vartheta_{H C, i, k}$ are not informative. Therefore, let us consider the likelihood function averaged by these parameters:

$$
\begin{aligned}
& p\left(Y_{k, 1}^{k+1} \mid X_{k}\right) \\
& =\frac{1}{2 \pi} \int_{-\pi}^{\pi} \ldots \int_{-\pi}^{\pi} p\left(Y_{k, 1}^{k+1} \mid X_{k}, \varphi_{0, i, k} \vartheta_{\mathrm{HC}, i, k}, i=\overline{1, n}\right) d \varphi_{0,1, k} \ldots d \varphi_{0, n, k}=v
\end{aligned}
$$

where, $I_{0}(x)$ is the zero-order Bessel function of imaginary argument:

$U_{i}^{2}\left(\alpha_{k}\right)=U_{c, i}^{2}\left(\alpha_{k}\right)+U_{s, i}^{2}\left(\alpha_{k}\right)$ 


$$
\begin{aligned}
& U_{c, i}\left(\alpha_{k}\right)=\frac{A}{\sigma_{n}^{2}} \sum_{l=1}^{M}\left[\begin{array}{l}
y_{A, i}\left(t_{k-1, l}\right) h_{\text {सKki }}\left(t-\tau_{A, i, k}\right) \\
\cos \left(\omega_{0} t_{k, l}+\left(\omega_{a i, k}+\omega_{A, \psi, i, k}\right)(l-1) T_{d}+\psi_{A, i}\left(\alpha_{k}\right)\right)
\end{array}\right. \\
& \left.+y_{B, i}\left(t_{k, l}\right) h_{\not k}\left(t-\tau_{B, i, k}\right) \cos \left(\begin{array}{l}
\omega_{0} t_{k, l}+\left(\omega_{\not i, k}-\omega_{B, \psi, i, k}\right) \\
(l-1) T_{d}+\psi_{B, i}\left(\alpha_{k}\right)
\end{array}\right)\right) \\
& \left.\left.+y_{C, i}\left(t_{k, l}\right) h_{\text {дk }}\left(t-\tau_{C, i, k}\right) \cos \left(\begin{array}{l}
\left.\omega_{0} t_{k, l}+\left(\omega_{a i, k}+\omega_{C, \psi, i, k}\right)\right) \\
(l-1) T_{d}+\psi_{C, i, k}\left(\alpha_{k}\right)
\end{array}\right)\right)\right] \\
& \left.\left.+y_{C, i}\left(t_{k, l}\right) h_{\text {дк }}\left(t-\tau_{C, i, k}\right) \cos \left(\begin{array}{l}
\omega_{0} t_{k, l}+\left(\omega_{a i, k}+\omega_{C, \psi, i, k}\right) \\
(l-1) T_{d}+\psi_{C, i, k}\left(\alpha_{k}\right)
\end{array}\right)\right)\right] \\
& U_{s, i}\left(\alpha_{k}\right)=\frac{A}{\sigma_{n}^{2}} \sum_{l=1}^{M}\left[\begin{array}{l}
y_{A, i}\left(t_{k, l}\right) h_{\pi k i}\left(t-\tau_{A, i, k}\right) \\
\sin \left(\omega_{0} t_{k, l}+\left(\omega_{a i, k}+\omega_{A, \psi, i, k}\right)(l-1) T_{d}+\psi_{A, i, k}\left(\alpha_{k}\right)\right)
\end{array}\right. \\
& \left.+y_{B, i}\left(t_{k, l}\right) h_{\text {д火 }}\left(t-\tau_{B, i, k}\right) \sin \left(\begin{array}{l}
\omega_{0} t_{k, l}+\left(\omega_{д i, k}-\omega_{B, \psi, i, k}\right) \\
(l-1) T_{d}+\psi_{B, i, k}\left(\alpha_{k}\right)
\end{array}\right)\right)+ \\
& \left.\left.+y_{C, i}\left(t_{k, l}\right) h_{\mathrm{dk}}\left(t-\tau_{C, i, k}\right) \sin \left(\begin{array}{l}
\left.\omega_{0} t_{k, l}+\left(\omega_{z i, k}-\omega_{C, \psi i, i, k}\right)\right) \\
(l-1) T_{d}+\psi_{C, i, k}\left(\alpha_{k}\right)
\end{array}\right)\right)\right]
\end{aligned}
$$

Let us represent (10) in the form of:

$$
\begin{aligned}
& U_{c, i}\left(\alpha_{k}\right)=I_{A i, k} \cos \left(\psi_{A i, k}\left(\alpha_{k}\right)\right)+I_{B i, k} \cos \left(\psi_{B i, k}\left(\alpha_{k}\right)\right) \\
& +I_{C i, k} \cos \left(\psi_{C i, k}\left(\alpha_{k}\right)\right)-\left(\begin{array}{l}
Q_{A i, k} \sin \left(\psi_{A i, k}\left(\alpha_{k}\right)\right)+Q_{B i, k} \sin \\
\left(\psi_{B i, k}\left(\alpha_{k}\right)\right)+Q_{C i, k} \sin \left(\psi_{C i, k}\left(\alpha_{k}\right)\right)
\end{array}\right) \\
& U_{s, i}\left(\alpha_{k}\right)=Q_{A i, k} \cos \left(\psi_{A i, k}\left(\alpha_{k}\right)\right)+Q_{B i, k} \cos \left(\psi_{B i, k}\left(\alpha_{k}\right)\right)+Q_{C i, k} \\
& \cos \left(\psi_{C i, k}\left(\alpha_{k}\right)\right)+\left(\begin{array}{l}
I_{A i, k} \sin \left(\psi_{A i, k}\left(\alpha_{k}\right)\right)+I_{B i, k} \sin \left(\psi_{B i, k}\left(\alpha_{k}\right)\right) \\
+I_{C i, k} \sin \left(\psi_{C i, k}\left(\alpha_{k}\right)\right)
\end{array}\right)
\end{aligned}
$$

where:

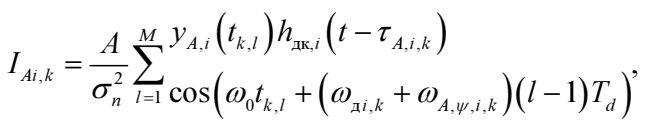

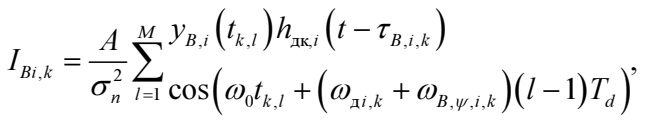

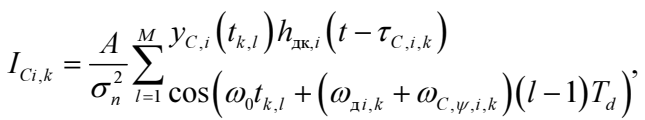

$$
\begin{aligned}
& Q_{A i, k}=\frac{A}{\sigma_{n}^{2}} \sum_{l=1}^{M} \sin \left(\omega_{0} t_{k, l}+\left(\omega_{a i, k}+\omega_{A, \psi, i, k}\right)(l-1) T_{d}\right)^{\prime}, \\
& Q_{B i, k}=\frac{A}{\sigma_{n}^{2}} \sum_{l=1}^{M} \sin \left(\omega_{0} t_{k, l}+\left(\omega_{\text {дi, }}+\omega_{B, \psi, i, k}\right)(l-1) T_{d}\right)^{\prime}, \\
& Q_{C i, k}=\frac{A}{\sigma_{n}^{2}} \sum_{l=1}^{M} \sin \left(\omega_{0} t_{k, l}+\left(\omega_{z i, k}+\omega_{C, \psi, i, k}\right)(l-1) T_{d}\right)
\end{aligned}
$$

Describe the multi-channel correlator, during the implementation of which, the Doppler frequencies $\omega_{a i, k}$, $\omega_{A, \psi, i, k}, \quad \omega_{B, \psi, i, k}, \quad \omega_{C, \psi, i, k}$ shall be replaced by the corresponding estimations $\tilde{\omega}_{\not i, k}, \tilde{\omega}_{A, \psi, i, k}, \tilde{\omega}_{B, \psi, i, k}, \tilde{\omega}_{C, \psi, i, k}$, which are formed in independent tracking rings such as those described in (Perov and Kharisov, 2010).

Let us note that the correlation integrals (11) shall be calculated in a single timeline.

Substituting (9) into (7) and performing the necessary transformations, we obtain the following:

$$
\begin{aligned}
& U_{i}^{2}\left(\alpha_{k}\right)=I_{A i, k}^{2}+Q_{A i, k}^{2}+I_{B i, k}^{2}+Q_{B i, k}^{2}+I_{C i, k}^{2}+Q_{C i, k}^{2}+ \\
& +2 \cos \left(\psi_{A i, k}\left(\alpha_{k}\right)-\psi_{B i, k}\left(\alpha_{k}\right)\right)\left(I_{A i, k} I_{B i, k}+Q_{A i, k} Q_{B i, k}\right)+ \\
& +2 \sin \left(\psi_{A i, k}\left(\alpha_{k}\right)-\psi_{B i, k}\left(\alpha_{k}\right)\right)\left(I_{A i, k} Q_{B i, k}-Q_{A i, k} I_{B i, k}\right)+ \\
& +2 \cos \left(\psi_{A i, k}\left(\alpha_{k}\right)-\psi_{C i, k}\left(\alpha_{k}\right)\right)\left(I_{A i, k} I_{C i, k}+Q_{A i, k} Q_{C i, k}\right)+ \\
& +2 \sin \left(\psi_{A i, k}\left(\alpha_{k}\right)-\psi_{C i, k}\left(\alpha_{k}\right)\right)\left(I_{A i, k} Q_{C i, k}-Q_{A i, k} I_{C i, k}\right)+ \\
& +2 \cos \left(\psi_{B i, k}\left(\alpha_{k}\right)-\psi_{C i, k}\left(\alpha_{k}\right)\right)\left(I_{B i, k} I_{C i, k}+Q_{B i, k} Q_{C i, k}\right)+ \\
& +2 \sin \left(\psi_{B i, k}\left(\alpha_{k}\right)-\psi_{C i, k}\left(\alpha_{k}\right)\right)\left(I_{B i, k} Q_{C i, k}-Q_{B i, k} I_{C i, k}\right)
\end{aligned}
$$

Let us introduce the vector:

$$
\psi=\left|\begin{array}{lllllllll}
\psi_{A 1} & \ldots & \psi_{A n} & \psi_{B 1} & \ldots & \psi_{B n} & \psi_{C 1} & \ldots & \psi_{C n}
\end{array}\right|^{\mathrm{T}}
$$

And a matrix $c$ such that $\alpha=c X$.

Let us transform the derivative:

$\frac{\partial F_{k}\left(X_{k}\right)}{\partial X}=\frac{\partial F_{k}\left(\psi\left(\alpha_{k}\right)\right)}{\partial \psi} \frac{\partial \psi}{\partial \alpha} \frac{\partial \alpha_{k}}{\partial X}=\frac{\partial F_{k}\left(\alpha_{k}\right)}{\partial \psi} \frac{\partial \psi}{\partial \alpha} c$

Let us consider the following:

$$
\begin{aligned}
& \frac{\partial F_{k}\left(\psi\left(\alpha_{k}\right)\right)}{\partial \psi}=\frac{\partial}{\partial \psi}\left[\sum_{i=1}^{n}\left(\ln I_{0}\left(X_{i}\left(\alpha_{k}\right)\right)\right)\right] \\
& =\left|\begin{array}{l}
\frac{\partial}{\partial \psi_{A 1}}\left(\ln I_{0}\left(U_{1}\left(\alpha_{k}\right)\right)\right) \\
\cdots \frac{\partial}{\partial \psi_{B 1}}\left(\ln I_{0}\left(U_{n}\left(\alpha_{k}\right)\right)\right) \cdots \frac{\partial}{\partial \psi_{C n}}\left(\ln I_{0}\left(U_{n}\left(\alpha_{k}\right)\right)\right)
\end{array}\right|
\end{aligned}
$$

Here:

$$
\frac{\partial}{\partial \psi_{A(B, C) i}}\left(\ln I_{0}\left(U_{i}\left(\alpha_{k}\right)\right)\right)=\frac{I_{1}\left(U_{i}\left(\alpha_{k}\right)\right)}{I_{0}\left(U_{i}\left(\alpha_{k}\right)\right)} \frac{\partial U_{i}\left(\alpha_{k}\right)}{\partial \psi_{A(B, C) i}}
$$

Is phase difference discriminator.

The derivative $\frac{\partial U_{i}\left(\alpha_{k}\right)}{\partial \psi_{A(B, C) i}}$ is obtained by differentiating (12) with respect to the corresponding parameter $\psi_{A(B, C) i}$ and, for example, for $\psi_{A i}$ it has the following form: 


$$
\begin{aligned}
& \frac{\partial U_{i}\left(\alpha_{k}\right)}{\partial \psi_{A i}}=\frac{1}{2 U_{i}(\alpha)} \frac{\partial U_{i}^{2}\left(\alpha_{k}\right)}{\partial \psi_{A i}} \\
& =\frac{1}{U_{i}\left(\alpha_{k}\right)}\left[-\sin \left(\psi_{A i, k}\left(\alpha_{k}\right)-\psi_{B i, k}\left(\alpha_{k}\right)\right)\left(I_{A i, k} I_{B i, k}+Q_{A i, k} Q_{B i, k}\right)+\right. \\
& +\cos \left(\psi_{A i, k}\left(\alpha_{k}\right)-\psi_{B i, k}\left(\alpha_{k}\right)\right)\left(I_{A i, k} Q_{B i, k}-Q_{A i, k} I_{B i, k}\right)- \\
& -\sin \left(\psi_{A i, k}\left(\alpha_{k}\right)-\psi_{C i, k-1}\left(\alpha_{k}\right)\right)\left(I_{A i, k} I_{C i, k}+Q_{A i, k} Q_{C i, k}\right)+ \\
& \left.+\cos \left(\psi_{A i, k}\left(\alpha_{k}\right)-\psi_{C i, k}\left(\alpha_{k}\right)\right)\left(I_{A i, k} Q_{C i, k}-Q_{A i, k} I_{C i, k}\right)\right]
\end{aligned}
$$

Let us write the phases $\psi_{A, i}\left(\alpha_{k}\right), \psi_{B, i}\left(\alpha_{k}\right), \psi_{C, i}\left(\alpha_{k}\right)$ with regard to (1), (4) in the following form:

$$
\begin{aligned}
& \psi_{A, i}\left(\alpha_{k}\right)=\frac{2 \pi L_{A O_{\mathrm{c}}} \mu_{\mathrm{HC}, i}^{\mathrm{T}} U_{\mathrm{c}}^{3}\left(\alpha_{k}\right) l_{A O_{\mathrm{c}}, \mathrm{c}}}{\lambda}, \\
& \psi_{B, i}\left(\alpha_{k}\right)=\frac{2 \pi L_{B O_{\mathrm{c}}} \mu_{\mathrm{HC}, i}^{\mathrm{T}} U_{\mathrm{c}}^{3}\left(\alpha_{k}\right) l_{B O_{\mathrm{c}}, \mathrm{c}}}{\lambda}, \\
& \psi_{c, i}\left(\alpha_{k}\right)=\frac{2 \pi L_{C O_{\mathrm{c}}} \mu_{\mathrm{HC}, i}^{\mathrm{T}} U_{\mathrm{c}}^{3}\left(\alpha_{k}\right) l_{C O_{\mathrm{c}}, \mathrm{c}}}{\lambda}
\end{aligned}
$$

Let us represent the vector $\psi$ in the form $\psi=\left|\begin{array}{lll}\psi_{A}^{\mathrm{T}} & \psi_{B}^{\mathrm{T}} & \psi_{C}^{\mathrm{T}}\end{array}\right|^{\mathrm{T}}$, where $\quad \psi_{A(B, C)}=\left|\begin{array}{lll}\psi_{A 1} & \ldots & \psi_{A n}\end{array}\right|^{\mathrm{T}} \quad$ and write the derivative $\frac{\partial \psi}{\partial \alpha}$ as a block matrix:

$$
\frac{\partial \psi}{\partial \alpha}=\left|\begin{array}{c}
\frac{\partial \psi_{A}}{\partial \alpha} \\
\frac{\partial \psi_{B}}{\partial \alpha} \\
\frac{\partial \psi_{C}}{\partial \alpha}
\end{array}\right|
$$

Where:

$$
\frac{\partial \psi_{A}}{\partial \alpha}=\left|\begin{array}{c}
\frac{\partial \psi_{A 1}}{\partial \alpha} \\
\ldots \\
\frac{\partial \psi_{A n}}{\partial \alpha}
\end{array}\right| \frac{\partial \psi_{B}}{\partial \alpha}=\left|\begin{array}{c}
\frac{\partial \psi_{B 1}}{\partial \alpha} \\
\ldots \\
\frac{\partial \psi_{B n}}{\partial \alpha}
\end{array}\right| \frac{\partial \psi_{C}}{\partial \alpha}=\left|\begin{array}{c}
\frac{\partial \psi_{C 1}}{\partial \alpha} \\
\ldots \\
\frac{\partial \psi_{C n}}{\partial \alpha}
\end{array}\right|
$$

Let us consider the derivative:

$$
\frac{\partial \psi_{A i}}{\partial \alpha}=\frac{2 \pi L_{A O_{\mathrm{c}}} \mu_{\mathrm{HC}, i}^{\mathrm{T}}}{\lambda} \frac{\partial}{\partial \alpha}\left(U_{\mathrm{c}}^{3}\left(\alpha_{k}\right) l_{A O_{c}, \mathrm{c}}\right)
$$

Let us introduce the matrix:

$$
M=\left|\begin{array}{c}
\mu_{\mathrm{HC}, 1}^{\mathrm{T}} \\
\mu_{\mathrm{HC}, 2}^{\mathrm{T}} \\
\cdots \\
\mu_{\mathrm{HC}, n}^{\mathrm{T}}
\end{array}\right|
$$

Then in view of (14), (15), the following expression can be written:

$\frac{\partial \psi_{A}}{\partial \alpha}=\frac{2 \pi L_{A O_{c}}}{\lambda} M \cdot \frac{\partial}{\partial \alpha}\left(U_{\mathrm{c}}^{3}\left(\alpha_{k}\right) l_{A O_{\mathrm{c}, \mathrm{c}}}\right)$

Similar expressions can be written for derivatives:

$$
\begin{aligned}
& \frac{\partial \psi_{B}}{\partial \alpha}=\frac{2 \pi L_{B O_{\mathrm{c}}}}{\lambda} M \cdot \frac{\partial}{\partial \alpha}\left(U_{\mathrm{c}}^{3}\left(\alpha_{k}\right) l_{B O_{\mathrm{c}}, \mathrm{c}}\right), \\
& \frac{\partial \psi_{C}}{\partial \alpha}=\frac{2 \pi L_{C O_{\mathrm{c}}}}{\lambda} M \cdot \frac{\partial}{\partial \alpha}\left(U_{\mathrm{c}}^{3}\left(\alpha_{k}\right) l_{C O_{\mathrm{e}}, \mathrm{c}}\right)
\end{aligned}
$$

Let us note that in (16), (17) differentiation with respect to the orientation angles $\alpha$ shall be applied only to elements of the transformation matrix $U_{\mathrm{c}}^{3}(\alpha)$, e.g.:

$\frac{\partial U_{\mathrm{c}}^{3}(\alpha)}{\partial \alpha_{1}}=\left|\begin{array}{ccc}\cos \left(\alpha_{3}\right) & -\sin \left(\alpha_{3}\right) & 0 \\ \sin \left(\alpha_{3}\right) & \cos \left(\alpha_{3}\right) & 0 \\ 0 & 0 & 1\end{array}\right|$

$\left|\begin{array}{ccc}\cos \left(\alpha_{2}\right) & 0 & \sin \left(\alpha_{2}\right) \\ 0 & 1 & 0 \\ -\sin \left(\alpha_{2}\right) & 0 & \cos \left(\alpha_{2}\right)\end{array}\right| \cdot\left|\begin{array}{ccc}0 & 0 & 0 \\ 0 & -\sin \left(\alpha_{1}\right) & -\cos \left(\alpha_{1}\right) \\ 0 & \cos \left(\alpha_{1}\right) & -\sin \left(\alpha_{1}\right)\end{array}\right|$

$\frac{\partial U_{\mathrm{c}}^{3}(\alpha)}{\partial \alpha_{2}}=\left|\begin{array}{ccc}\cos \left(\alpha_{3}\right) & -\sin \left(\alpha_{3}\right) & 0 \\ \sin \left(\alpha_{3}\right) & \cos \left(\alpha_{3}\right) & 0 \\ 0 & 0 & 1\end{array}\right|$

$\left|\begin{array}{ccc}-\sin \left(\alpha_{2}\right) & 0 & \cos \left(\alpha_{2}\right) \\ 0 & 0 & 0 \\ -\cos \left(\alpha_{2}\right) & 0 & -\sin \left(\alpha_{2}\right)\end{array}\right| \cdot\left|\begin{array}{ccc}1 & 0 & 0 \\ 0 & \cos \left(\alpha_{1}\right) & -\sin \left(\alpha_{1}\right) \\ 0 & \sin \left(\alpha_{1}\right) & \cos \left(\alpha_{1}\right)\end{array}\right|$,

$\frac{\partial U_{\mathrm{c}}^{3}(\alpha)}{\partial \alpha_{3}}=\left|\begin{array}{ccc}-\sin \left(\alpha_{3}\right) & -\cos \left(\alpha_{3}\right) & 0 \\ \cos \left(\alpha_{3}\right) & -\sin \left(\alpha_{3}\right) & 0 \\ 0 & 0 & 0\end{array}\right|$

$\cdot\left|\begin{array}{ccc}\cos \left(\alpha_{2}\right) & 0 & \sin \left(\alpha_{2}\right) \\ 0 & 1 & 0 \\ -\sin \left(\alpha_{2}\right) & 0 & \cos \left(\alpha_{2}\right)\end{array}\right| \cdot\left|\begin{array}{ccc}1 & 0 & 0 \\ 0 & \cos \left(\alpha_{1}\right) & -\sin \left(\alpha_{1}\right) \\ 0 & \sin \left(\alpha_{1}\right) & \cos \left(\alpha_{1}\right)\end{array}\right|$

In view of the obtained expressions, let us write:

$$
\frac{\partial \psi}{\partial \alpha}=\frac{2 \pi}{\lambda}\left|\begin{array}{l}
L_{A O_{\mathrm{c}}} M \cdot \frac{\partial}{\partial \alpha}\left(U_{\mathrm{c}}^{3}\left(\alpha_{k}\right) l_{A O_{\mathrm{c}, \mathrm{c}}}\right) \\
L_{B O_{\mathrm{c}}} M \cdot \frac{\partial}{\partial \alpha}\left(U_{\mathrm{c}}^{3}\left(\alpha_{k}\right) l_{B O_{\mathrm{c}, \mathrm{c}}}\right) \\
L_{C O_{\mathrm{c}}} M \cdot \frac{\partial}{\partial \alpha}\left(U_{\mathrm{c}}^{3}\left(\alpha_{k}\right) l_{C O_{\mathrm{c}, \mathrm{c}} \mathrm{c}}\right)
\end{array}\right|
$$

Then, let us represent (17) in the form of: 


$$
\frac{\partial F_{k}\left(X_{k}\right)}{\partial X}=\frac{2 \pi}{\lambda} \frac{\partial F_{k}\left(\alpha_{k}\right)}{\partial \psi}\left|\begin{array}{l}
L_{A O_{\mathrm{c}}} M \cdot \frac{\partial}{\partial \alpha}\left(U_{\mathrm{c}}^{3}\left(\alpha_{k}\right) l_{A O_{\mathrm{c}}, \mathrm{c}}\right) \\
L_{B O_{\mathrm{c}}} M \cdot \frac{\partial}{\partial \alpha}\left(U_{\mathrm{c}}^{3}\left(\alpha_{k}\right) l_{B O_{\mathrm{c}}, \mathrm{c}}\right) \\
L_{C O_{\mathrm{c}}} M \cdot \frac{\partial}{\partial \alpha}\left(U_{\mathrm{c}}^{3}\left(\alpha_{k}\right) l_{C O_{\mathrm{c}}, \mathrm{c}}\right)
\end{array}\right| c
$$

where, the matrix $c$ is determined by the ratio $\alpha=c X$, i.e., $c=\left|I_{3 \times 3} 0_{3 \times 3}\right|$, where $I_{3 \times 3}$ is the identity matrix of $3 \times 3$ size.

Substituting (18) into (5), let us write an equation for estimating the state vector:

$$
\begin{aligned}
& \hat{X}_{k}=\tilde{X}_{k}+D_{X, k} c^{\mathrm{T}}\left[\frac{2 \pi}{\lambda}\left|\begin{array}{l}
L_{A O_{\mathrm{c}}} M \cdot \frac{\partial}{\partial \alpha}\left(U_{\mathrm{c}}^{3}\left(\alpha_{k}\right) l_{A O_{\mathrm{c}}, \mathrm{c}}\right) \\
L_{B O_{\mathrm{c}}} M \cdot \frac{\partial}{\partial \alpha}\left(U_{\mathrm{c}}^{3}\left(\alpha_{k}\right) l_{B O_{\mathrm{c}}, \mathrm{c}}\right) \\
L_{C O_{\mathrm{c}}} M \cdot \frac{\partial}{\partial \alpha}\left(U_{\mathrm{c}}^{3}\left(\alpha_{k}\right) l_{C O_{\mathrm{c}}, \mathrm{c}}\right)
\end{array}\right|\left(\frac{\partial F_{k}\left(\alpha_{k}\right)}{\partial \psi}\right)^{\mathrm{T}}\right] \\
& =\tilde{X}_{k}+D_{X, k} c^{\mathrm{T}}\left[\frac{2 \pi}{\lambda}\left|\begin{array}{l}
L_{A O_{\mathrm{c}}} M \cdot \frac{\partial}{\partial \alpha}\left(U_{\mathrm{c}}^{3}\left(\alpha_{k}\right) l_{A O_{\mathrm{c}}, \mathrm{c}}\right) \\
L_{B O_{\mathrm{c}}} M \cdot \frac{\partial}{\partial \alpha}\left(U_{\mathrm{c}}^{3}\left(\alpha_{k}\right) l_{B O_{\mathrm{c}}, \mathrm{c}}\right) \\
L_{C O_{\mathrm{c}}} M \cdot \frac{\partial}{\partial \alpha}\left(U_{\mathrm{c}}^{3}\left(\alpha_{k}\right) l_{C O_{\mathrm{c}}, \mathrm{c}}\right)
\end{array}\right| u_{z \psi, k}\right]
\end{aligned}
$$

$=\tilde{X}_{k}+D_{X, k} c^{\mathrm{T}} u_{\text {да,k }}$

Where:

$$
u_{\text {dis } \psi, k}=\left(\frac{\partial F_{k}\left(\alpha_{k}\right)}{\partial \psi}\right)^{\mathrm{T}}
$$

Is the vector of discriminators of phase difference according to the signals of visible NS:

$$
u_{d i s \alpha, k}=\frac{2 \pi}{\lambda}\left|\begin{array}{l}
L_{A O_{\mathrm{c}}} M \cdot \frac{\partial}{\partial \alpha}\left(U_{\mathrm{c}}^{3}\left(\alpha_{k}\right) l_{A O_{\mathrm{c}}, \mathrm{c}}\right) \\
L_{B O_{\mathrm{c}}} M \cdot \frac{\partial}{\partial \alpha}\left(U_{\mathrm{c}}^{3}\left(\alpha_{k}\right) l_{B O_{\mathrm{c}}, \mathrm{c}}\right) \\
L_{C O_{\mathrm{c}}} M \cdot \frac{\partial}{\partial \alpha}\left(U_{\mathrm{c}}^{3}\left(\alpha_{k}\right) l_{C O_{\mathrm{c}}, \mathrm{c}}\right)
\end{array}\right| u_{d i s \psi, k}^{\mathrm{T}}
$$

Is the vector of discriminators for the orientation angles.

Generalized block diagram of a single-stage filtration system for orientation angles of the object is shown in Fig. 3.

Let us note that in the diagram of Fig. 3 has a multichannel correlator.

\section{The Potential Accuracy of the Estimation of Orientation Angles}

It is known (Perov and Kharisov, 2010) that the discriminator is a device for which the parameters of the received signal and their evaluation are constant at the duration of the interval $T$. To characterize the quality of the estimates generated in the optimal discriminator, one can use the lower limit of the Cramer-Rao for the dispersion of the parameter estimates. Let us calculate this boundary for the problem.

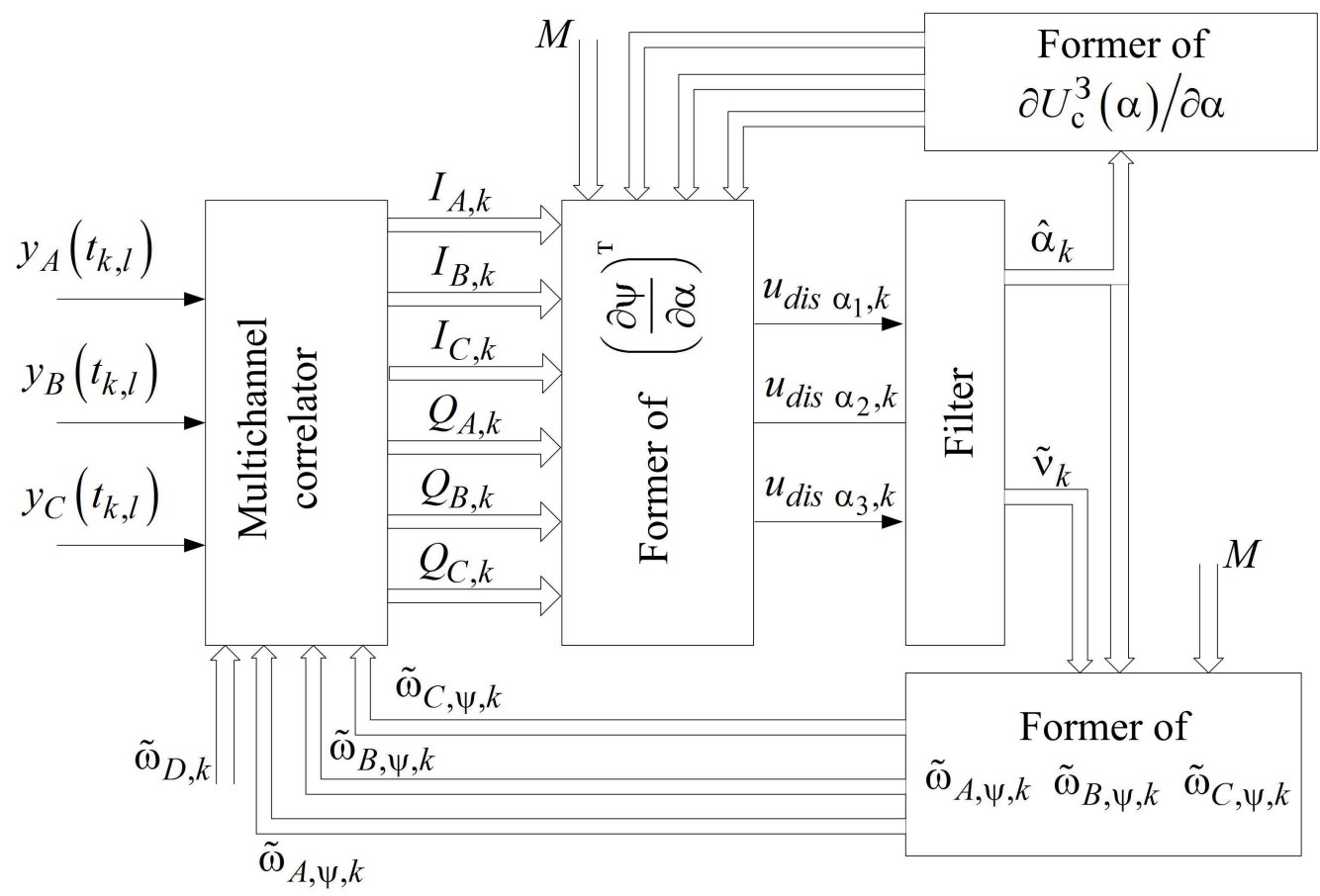

Fig. 3. Generalized block diagram of a single-stage filtration system for orientation angles of the object 
To simplify notations, let us turn to continuous time and consider the time interval $[0, T]$. Lower boundary for the matrix $D_{p o t, \alpha}$ of the dispersion of estimation errors of the orientation angles $\alpha$ is determined by the expression (Tikhonov and Kharisov, 2004; Perov, 2012):

$$
D_{p o t, a}=-J^{-1}
$$

where, $J$ is the Fisher's information matrix:

$$
J=E\left[\frac{\partial}{\partial \alpha}\left(\frac{\partial \ln \left(p\left(Y_{0}^{T} \mid \alpha\right)\right)}{\partial \alpha}\right)^{\mathrm{T}}\right]
$$

where, $p\left(Y_{0}^{T} \mid \alpha\right)$ is described by (8), $E\left[^{*}\right]$ is a sign of the mathematical expectation of a random variable in brackets.

Let us consider the derivative of the natural logarithm (7) by $\alpha$ :

$$
\frac{\partial \ln \left(p\left(Y_{0}^{T} \mid \alpha\right)\right)}{\partial \alpha}=\sum_{i=1}^{n} \frac{I_{1}\left(X_{i}(\alpha)\right)}{I_{0}\left(X_{i}(\alpha)\right)} \frac{\partial X_{i}(\alpha)}{\partial \alpha}
$$

where, $I_{1}(x)$ is the first order Bessel function of imaginary argument.

In the standard signal/noise ratio corresponding to the Interface Control Document (n.a., 2011) $\frac{I_{1}\left(X_{i}(\alpha)\right)}{I_{0}\left(X_{i}(\alpha)\right)} \approx 1$. Therefore, (21) takes the form of:

$$
\frac{\partial \ln \left(p\left(Y_{0}^{T} \mid \alpha\right)\right)}{\partial \alpha}=\sum_{i=1}^{n} \frac{\partial X_{i}(\alpha)}{\partial \alpha}
$$

Taking into account (22), let us write (20) in the form of:

$$
J=\sum_{i=1}^{n} M\left[\frac{\partial}{\partial \alpha}\left(\frac{\partial X_{i}(\alpha)}{\partial \alpha}\right)^{\mathrm{T}}\right]
$$

As $X_{i}$ is determined by synphase and quadrature components $I_{j, i}, Q_{j, i}$, then in order to perform the averaging in (23), it is necessary to have the mathematical expectations $I_{j, i}$ and $Q_{j, i}$. Therefore, let us perform averaging in (11):

$$
\begin{aligned}
& M\left[I_{j, i}\right]=2 q_{c / n_{0}} T \cos \left(\varphi_{0, i}+\psi_{A_{j}, i}(\alpha)\right), \\
& M\left[Q_{j, i}\right]=-2 q_{c / n_{0}} T \sin \left(\varphi_{0, i}+\psi_{A_{j}, i}(\alpha)\right)
\end{aligned}
$$

where, $q_{c} / n_{0}=P_{s} / N_{0}$ is the ratio of signal power to the power of spectral density of the internal noise.

Taking into account (24) and the relations (9) and (3), let us calculate $M\left[X_{i}\right]=6 q_{c} / n_{0} T$.

Performing in (23) differentiation with respect to $\alpha$ and averaging the result obtained by realizations of noise, taking into account (24), let us write the expression for the matrix elements $J$ :

$$
J_{v, \gamma}=-2 q_{c / n_{0}} T \sum_{i=1}^{n}\left[\begin{array}{c}
\left(\frac{\partial \psi_{A_{j}, i}(\alpha)}{\partial \alpha_{v}} \frac{\partial \psi_{A_{j}, i}(\alpha)}{\partial \alpha_{\gamma}}\right) \\
\sum_{j=1}^{3}-\frac{1}{3}\left(\sum_{j=1}^{m} \frac{\partial \psi_{A_{j}, i}(\alpha)}{\partial \alpha_{v}}\right)\left(\sum_{j=1}^{m} \frac{\partial \psi_{A_{j}, i}(\alpha)}{\partial \alpha_{\gamma}}\right)
\end{array}\right]
$$

where, $v=\overline{1,3}, \gamma=\overline{1,3}$ :

$$
\begin{aligned}
& \frac{\partial \psi_{A_{j}, i}(\alpha)}{\partial \alpha_{v}}=\frac{\partial}{\partial \alpha_{v}}\left(\frac{2 \pi L_{A_{j} O_{\mathrm{c}}} \mu_{\mathrm{HC}, i}^{\mathrm{T}}\left(U_{\mathrm{c}}^{3}(\alpha) l_{A_{j} O_{\mathrm{c}}, \mathrm{c}}\right)}{\lambda}\right) \\
& =\frac{2 \pi L_{A_{j} O_{\mathrm{c}}}}{\lambda} \mu_{\mathrm{HC}, i}^{\mathrm{T}}\left(\frac{\partial U_{\mathrm{c}}^{3}(\alpha)}{\partial \alpha_{v}} l_{A_{j} O_{\mathrm{c}}, \mathrm{c}}\right)=\frac{2 \pi L_{A_{j} O_{\mathrm{c}}}}{\lambda} \mu_{\mathrm{HC}, i}^{\mathrm{T}}\left(\frac{\partial U_{\mathrm{c}}^{3}(\alpha)}{\partial \alpha_{v}} l_{A_{j} O_{\mathrm{c}}, \mathrm{c}}\right)
\end{aligned}
$$

Let us consider the special case where the distance $L_{A_{j} O_{\mathrm{c}}}$, from the point $O_{c}$ to all points of reception $A_{j}$, $j=\overline{1, m}$ are the same and equal to $l$. Then (25) can be written as follows:

$$
\begin{aligned}
& J_{v, \gamma}=-2 q_{c / n_{0}} T\left(\frac{2 \pi l}{\lambda}\right)^{2} \\
& \times \sum_{i=1}^{n}\left[\sum_{j=1}^{m}-\frac{\partial \tilde{\psi}_{A_{j}, i}(\alpha)}{\partial \alpha_{v}} \frac{\partial \tilde{\psi}_{A_{j}, i}(\alpha)}{\partial \alpha_{\gamma}}\right) \\
& \left.\left.-\sum_{j=1}^{m} \frac{\partial \tilde{\psi}_{A_{j}, i}(\alpha)}{\partial \alpha_{v}}\right)\left(\sum_{j=1}^{m} \frac{\partial \tilde{\psi}_{A_{j}, i}(\alpha)}{\partial \alpha_{\gamma}}\right)\right]
\end{aligned}
$$

where:

$$
\frac{\partial \tilde{\psi}_{A_{j}, i}(\alpha)}{\partial \alpha_{v}}=\mu_{\mathrm{HC}, i}^{\mathrm{T}}\left(\frac{\partial U_{\mathrm{c}}^{3}(\alpha)}{\partial \alpha_{v}} l_{A_{j} O_{\mathrm{c}}, \mathrm{c}}\right)
$$

From (26) it follows that the elements $J_{v, \gamma}$ of the Fisher's information matrix contain the same factor $2 q_{c / n_{0}} T\left(\frac{2 \pi l}{\lambda}\right)^{2}$, i.e., it is possible to write the following:

$J=-2 q_{c / n_{0}} T\left(\frac{2 \pi l}{\lambda}\right)^{2} \tilde{J}$

Where: 


$$
\tilde{J}=\sum_{i=1}^{n}\left[\begin{array}{c}
\left(\frac{\partial \tilde{\psi}_{A_{j}, i}(\alpha)}{\partial \alpha_{v}} \frac{\partial \tilde{\psi}_{A_{j}, i}(\alpha)}{\partial \alpha_{\gamma}}\right) \\
\sum_{j=1}^{m}-\frac{1}{m}\left(\sum_{j=1}^{m} \frac{\partial \tilde{\psi}_{A_{j}, i}(\alpha)}{\partial \alpha_{v}}\right)\left(\sum_{j=1}^{m} \frac{\partial \tilde{\psi}_{A_{j}, i}(\alpha)}{\partial \alpha_{\gamma}}\right)
\end{array}\right]
$$

Substituting (27) into (19), let us write:

$$
D_{p o t, a}=\frac{1}{2 q_{c / n_{0}} T\left(\frac{2 \pi l}{\lambda}\right)^{2}} \tilde{J}^{-1}
$$

About (28) is the source of well-known provisions that the potential accuracy of the estimation of the orientation angle is as higher as better is the signal/noise ratio $q_{c} / n_{0} T$ and as higher is the ratio $l / \lambda$, which characterize the ratio of some characteristic linear dimension $l$ of the receiving system to the wavelength of the received navigation signal. About (28) also means that the potential accuracy of the estimation of orientation angles of the object by the NS signals is dependent on the spatial angle between the lines connecting the receiving points and the direction of arrival of navigation signals from the number $n$ of the processed NS signals and the number $m$ of reception points.

To analyse the expressions (19) and (25), let us use the following procedure. Let the values of the vector of orientation angles $\alpha$ be independent random variables that take values from a given range, for example $\alpha \in[-$ $\left.30^{\circ}, 30^{\circ}\right], \beta \in\left[-20^{\circ}, 20^{\circ}\right], \gamma \in\left[0^{\circ}, 360^{\circ}\right]$. We also believe the location of navigation satellites relatively to the object to be random with uniform distribution law in the upper hemisphere. We are interested in the histogram of distribution of the values of standard errors estimation of the angular orientation vector $D_{p o t, \alpha 1}, D_{p o t, \alpha 2}, D_{p o t, \alpha 3}(19)$.

Let us assume $q_{c} / n_{0}$, expressed in decibels $\tilde{q}_{c / n_{0}}=$ $45 \mathrm{dBHz}, T=5 \mathrm{~ms}, \lambda=c / f_{0}, f_{0}=1575.42 \mathrm{~Hz}$. Let us consider the 3 reception point located in the plane $O_{c} X_{c} Y_{c}$ and lying at the vertices of an equilateral triangle inscribed in a circle of radius $R=1 \mathrm{~m}$ centred at the point $O_{c}$.

Figures 4-6 show histograms of the distribution of values of standard errors estimation of different angles at the reception $n=4$ signals from navigation satellites.

Figures 5 and 6 show that the histogram distributions for the various components of the angular orientation are close enough to each other. Therefore, we will only talk about one component, for example $\alpha$. The most probable value of the mean square error of the angle estimation is $\sigma_{\alpha}=3.15$ arc. min., mean square scatter of values is $\sim 1$ arc. min.

Let us consider the effect of the number of navigation satellites for distribution histogram of mean square errors for angle estimation. Figures 7 and 8 show distribution histograms of values of mean square error of $\alpha$ angle estimations calculated at $n=10$ and $n=24$ and when receiving signals in the same three points.

From the histogram it is clear that with the increase of the number of NS processed signals, there is a decrease of the most probable value of the mean squared error of angle estimation $\left(\sigma_{\alpha}=3.15\right.$ arc. min. for $n=4$; $\sigma_{\alpha}=2$ arc. min. for $n=10 ; \sigma_{\alpha}=1.3 \mathrm{arc}$. $\min$. for $n=$ $24)$; the range of possible values of the mean square errors of the angle estimation is narrowed.

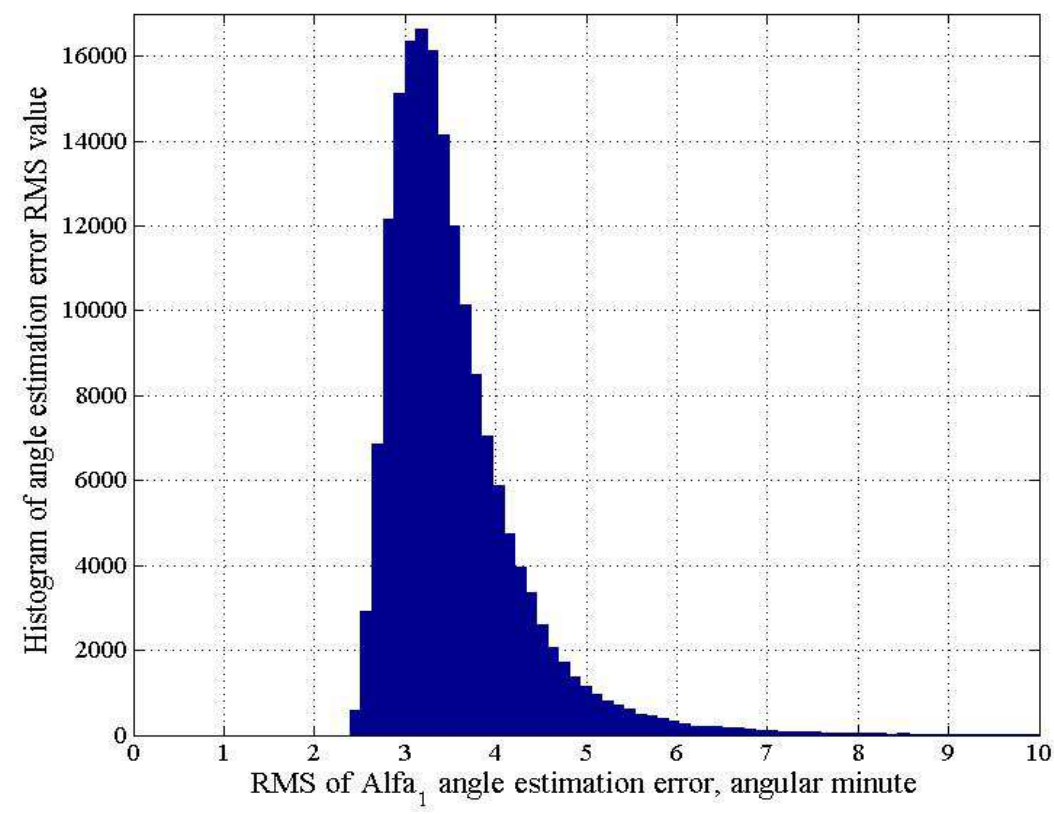

Fig. 4. Histogram of the distribution of values of mean square errors of estimation of the angle $\alpha_{1}$ at $n=4$ 


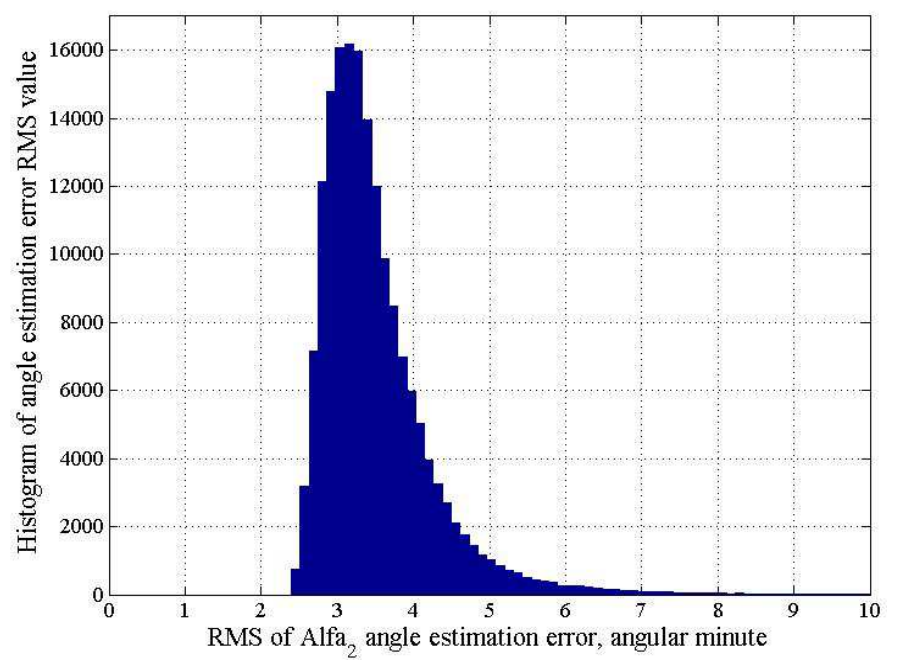

Fig. 5. Histogram of the distribution of values of mean square errors of estimation of the angle $\alpha_{2}$ at $n=4$

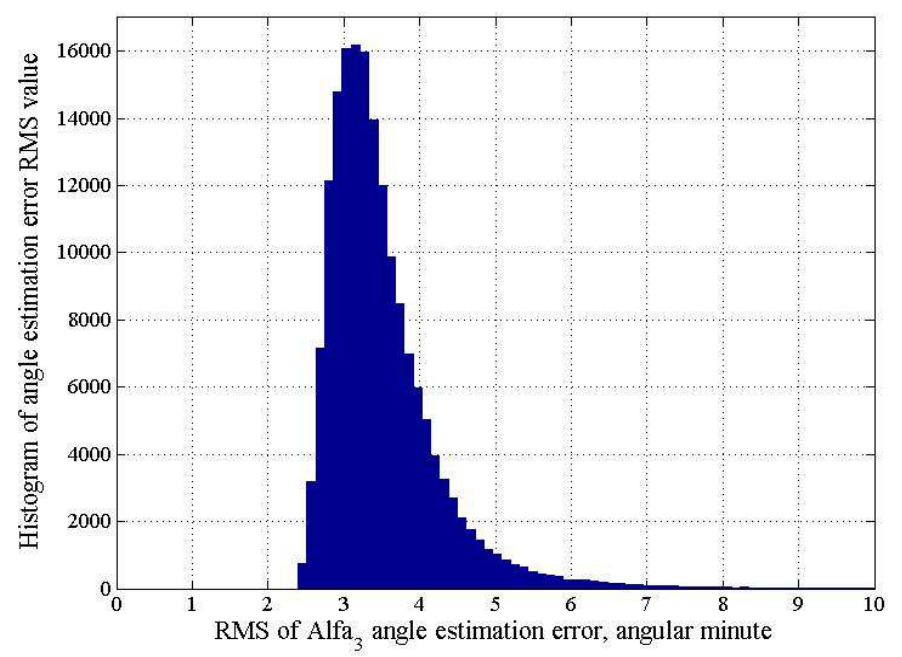

Fig. 6. Histogram of the distribution of values of mean square errors of estimation of the angle $\alpha_{3}$ at $n=4$

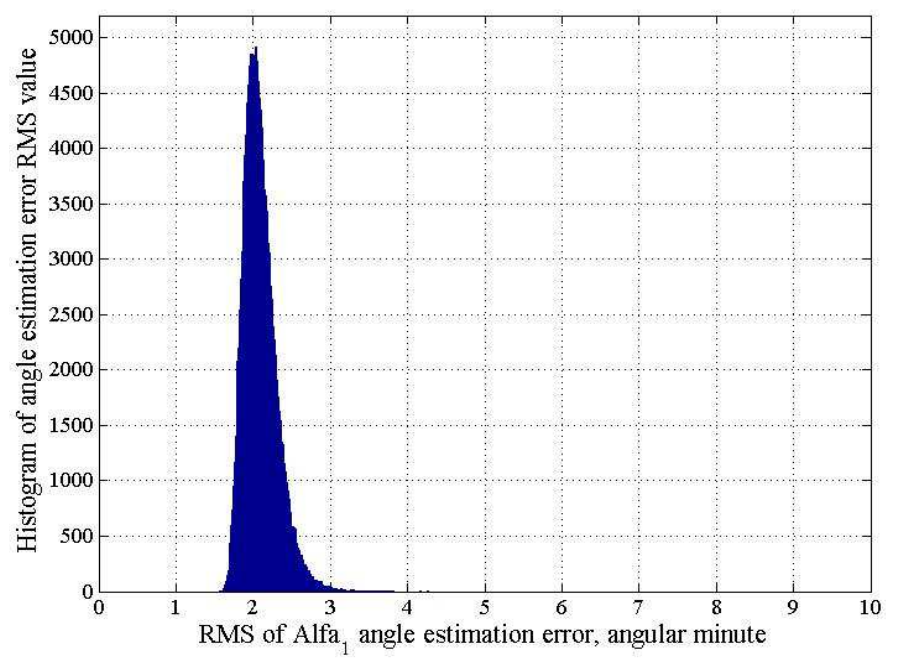

Fig. 7. Histogram of the distribution of values of mean square errors of estimation of the angle $\alpha_{1}$ at $n=10$ 


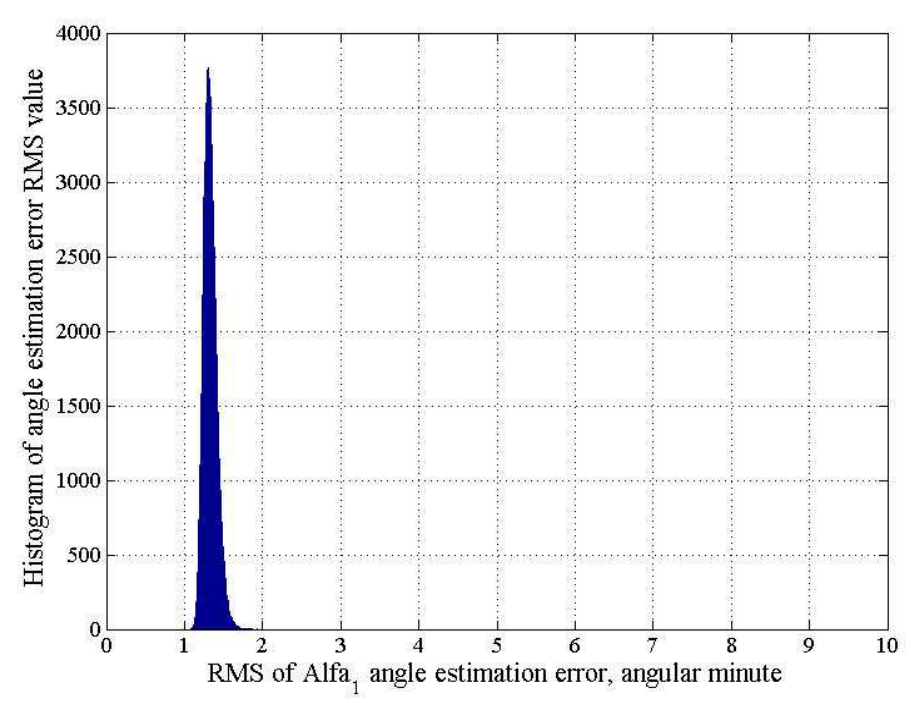

Fig. 8. Histogram of the distribution of values of mean square errors of estimation of the angle $\alpha_{1}$ at $n=24$

\section{Results}

\section{Simulation of the Synthesized Tracking System}

To carry out a simulation of the synthesized tracking system for orientation angles, let us simplify the dispersion Equations 6.

The essence of this simplification is to replace the derivative $\frac{\partial}{\partial X}\left(\frac{\partial \tilde{F}_{k}\left(\tilde{X}_{k}\right)}{\partial X}\right)^{T}$ with its mean value $\left.E\left[\frac{\partial}{\partial X}\left(\frac{\partial \tilde{F}_{k}\left(\tilde{X}_{k}\right)}{\partial X}\right)^{T}\right]\right|_{\tilde{X}_{k}=X_{k}}$. Carrying out this averaging, we obtain the following:

$$
\begin{aligned}
& \left.E\left[\frac{\partial}{\partial X}\left(\frac{\partial \tilde{F}_{k}\left(\tilde{X}_{k}\right)}{\partial X}\right)^{T}\right]\right|_{\tilde{X}_{k}=X_{k}} \\
& =\left.E\left[c^{\mathrm{T}} \frac{\partial}{\partial \tilde{\alpha}}\left(\frac{\partial \tilde{F}_{k}\left(\tilde{\alpha}_{k}\right)}{\partial \alpha}\right)^{T} c\right]\right|_{\tilde{\alpha}_{k}=\alpha_{k}}=c^{\mathrm{T}} J c
\end{aligned}
$$

Taking into account (29) and (19), the dispersion Equations 6 can be written as follows:

$$
\tilde{D}_{X, k}=F D_{X, k-1} F^{\mathrm{T}}+G D_{\xi} G^{\mathrm{T}}, D_{X, k}^{-1}=\tilde{D}_{x, k}^{-1}+c^{\mathrm{T}} D_{p o t, \alpha}^{-1} c
$$

Equation 30 is a matrix equation with constant coefficients, which solution in the steady state shall be denoted as $D_{X, \text { steady }}$.

Synthesized algorithm of optimal filtering for orientation angles of the object was simulated on a computer at a steady value of $D_{X, \text { steady }}$.

For clarity, we present the results of a simplified version. Assume that the triangle $A B C$ is isosceles with a side length of $l$, point $O_{c} \mathrm{CS} O_{c} X_{c} Y_{c} Z_{c}$ is located in the middle of the line $A B$, the axis $O_{c} X_{\mathrm{c}}$ is directed to the point $B$ and the axis $O_{c} Y_{c}$-to the point $C$. The object is rotated around the axis $O_{c} Y_{c}$ only.

In the simulation, we assume that $V_{0}=300 \mathrm{~m} \mathrm{~s}^{-1} ; \mathrm{a}_{0}$ $=40 \mathrm{~m} / \mathrm{s}^{2} ; v=\frac{2 \pi}{5 \times 60}, \tilde{q}_{c / n_{0}}=40 \mathrm{dBHz}, l=1.5 \mathrm{~m}$.

In a tracking system (20), we shall use a filter with constant parameters of gains:

$$
K_{1}=K_{1 \mathrm{H}} T, K_{2}=K_{2 \mathrm{H}} T, K_{2 \mathrm{H}}=\left(\Delta f_{\mathrm{cc}} / 0,53\right)^{2}, K_{1 \mathrm{H}}=\sqrt{2 K_{2 \mathrm{H}}}
$$

where, $\Delta f_{c c}$ is the bandwidth of the Tracking System (TS).

We assume that the errors on Doppler frequencies are not presented. Let us consider first the case of zero initial error in the angle and angular velocity. Assume that $\Delta f_{c c}$ $=2 \mathrm{~Hz}$ and the number of NS is 8 and is uniformly distributed angularly in sight. Figure 9 shows the implementation of the tracking error in the angle. Mean square error of the estimation is $\sigma \beta=0.75$ arc. min.

Let us narrow TS bandwidth $\Delta f_{c c}=0.5 \mathrm{~Hz}$. Figure 10 shows the implementation of the tracking error in the angle. Mean square error of the estimation is $\sigma \beta=0.34 \mathrm{arc}$. min.

Assume $\Delta f_{c c}=2 \mathrm{~Hz}$ and the number of navigation satellites is 2 (the first two of the preceding 8). Figure 11 shows the implementation of the tracking error in the angle. Mean square error of the estimation is $\sigma \beta=5.48$ arc. min.

Let us consider the effect of the initial error in the angular coordinate (with zero error in the angular velocity). Let us set the initial error in the angular coordinate $\Delta \beta_{\text {нач }}=5^{\circ}, \Delta f_{c c}=2 \mathrm{~Hz}$ and the number of navigation satellites is 8 . Figure 12 shows the implementation of the tracking error in the angle. Mean square error estimation of the angle in that mode is $\sigma \beta$ $=0.77$ arc. $\mathrm{min}$. 


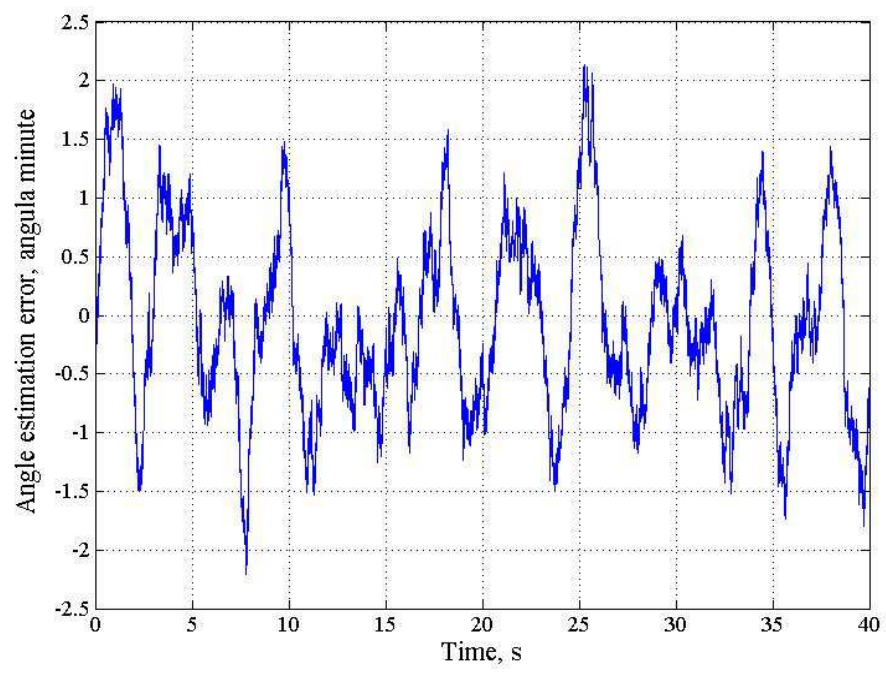

Fig. 9. Tracking error in the angle at $\Delta f_{c c}=2 \mathrm{~Hz}, n=8$

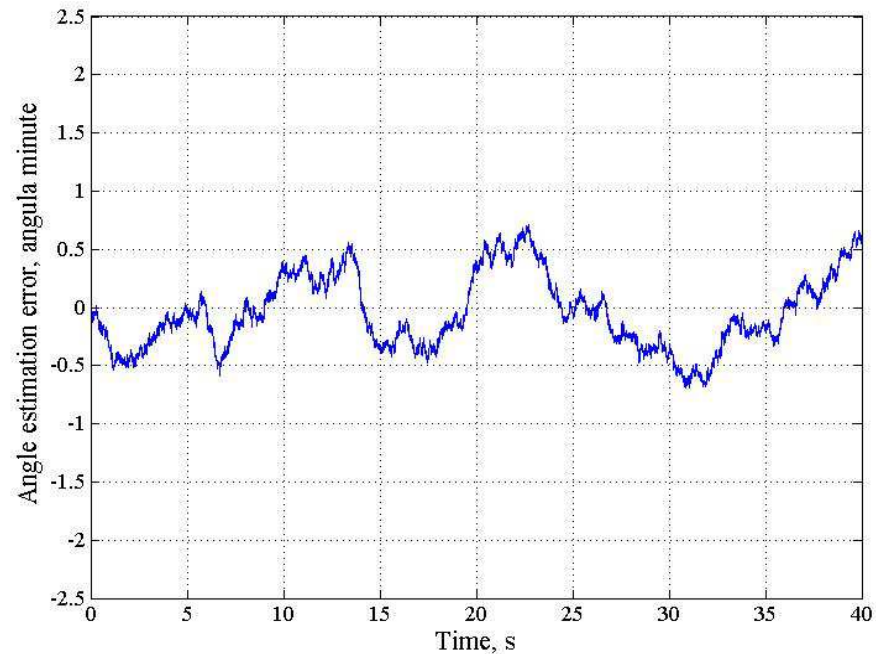

Fig. 10. Tracking error in the angle at $\Delta f_{c c}=0.5 \mathrm{~Hz}, n=8$

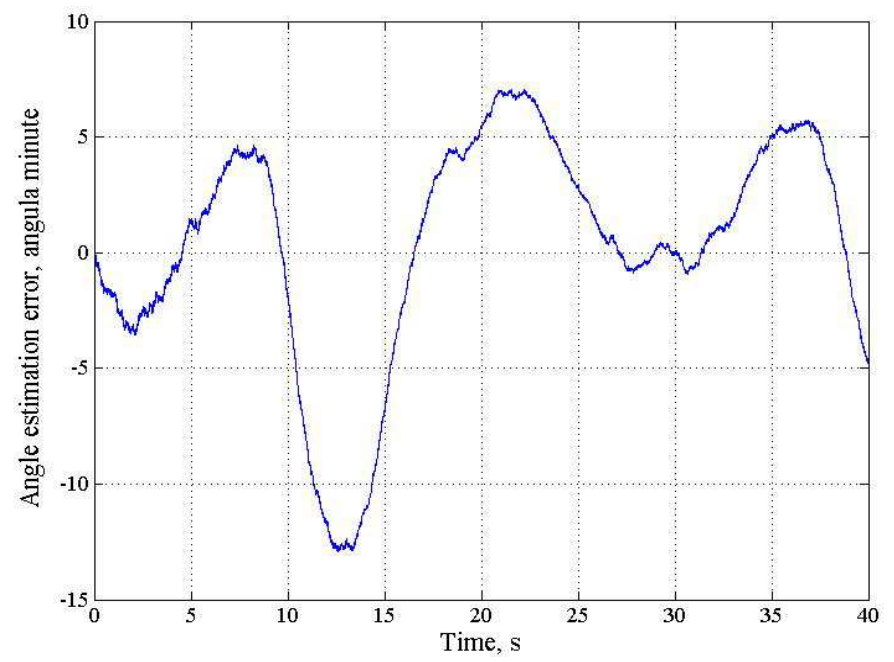

Fig. 11. Tracking error in the angle at $\Delta f_{c c}=2 \mathrm{~Hz}, n=2$ 


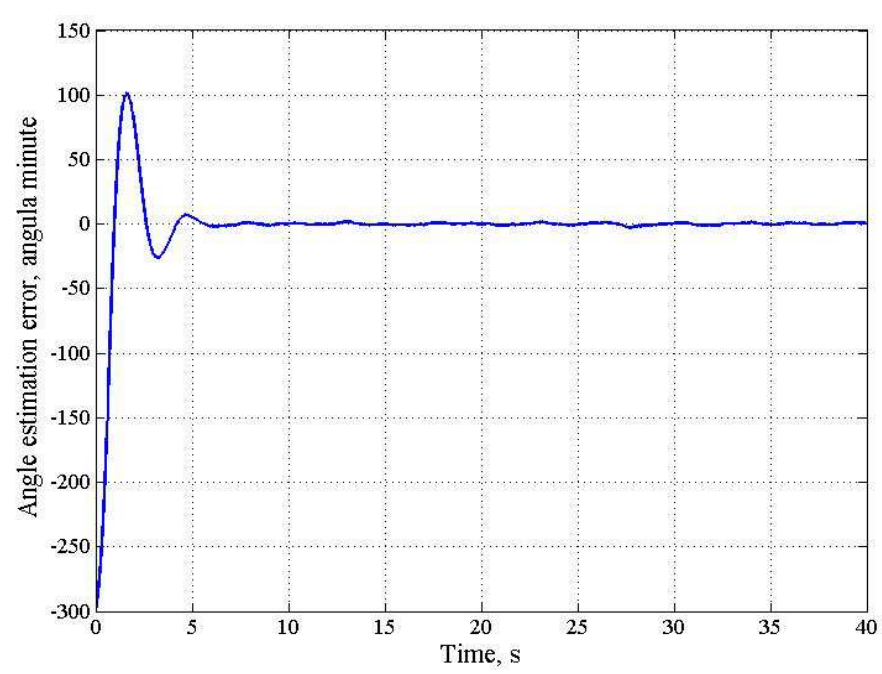

Fig. 12. Tracking error in the angle at $\Delta f_{c c}=2 \mathrm{~Hz}, n=8, \Delta \beta_{\text {нач }}=5 \mathrm{deg}$

\section{Discussion}

From the simulation results, it is clear that the synthesized filter algorithm for orientation angles of the object is successfully working in various tactical situations when the initial error on the orientation angles not exceeding 5 degrees. We also note the following:

- The synthesized estimation algorithm for orientation angles of the object has no tracking systems for the phase differences of the received signals, as was the case in the algorithm described in (Perov and Kharisov, 2010)

- The synthesized estimation algorithm of orientation angles of the object has no procedures for resolving ambiguity of the phase measurements on signals of navigation satellites

\section{Conclusion}

In this study, the theory of optimal filtering of information processes was used to synthesize the optimal one-stage algorithm for estimation of orientation angles of the object by the signals of satellite radio navigation system.

Equations of optimal filtering are obtained, as well as their representation in the form of a tracking system including a discriminator for orientation angles and a smoothing filter.

The synthesized algorithms have no tracking systems for the phases of the received signals (or phase differences) and procedures for resolving ambiguity of phase measurements, which enhances its noise immunity, while maintaining high precision estimation of orientation angles.

Expressions are obtained for the calculation of the lower limit of matrix of dispersion estimation errors for orientation angles of the object when receiving signals from an arbitrary number of navigation satellites in the interval with duration of $T$, in which the orientation angles do not change over the signals of satellite navigation systems with an arbitrary number of navigation signals.

Examples of receiving from 4 to 24 signals from navigation satellites at three points show that an increase of the received signals decreases the most probable value of the mean squared error for estimation of angles ( 3 to 1.2 arc minutes respectively at the signal/noise ratio of $45 \mathrm{dBHz}$ and $T=5 \mathrm{~ms}$ ). The range of possible values of the standard errors for angles estimation is narrowed. The results of simulation are provided, from which it follows that at the length of the spaced receiving points of $l=1.5 \mathrm{~m}$, there can be achieved a mean square error estimation of the orientation of angles in the steady state of $0.5 \ldots 0.7$ arc. $\min$.

\section{Acknowledgement}

The author thanks National Research University «Moscow Power Engineering Institute» for supporting this research.

\section{Ethics}

The authors have no conflicts of interest in the development and publication of current research.

\section{References}

Boriskin, A., A. Veytsel, V. Veytsel, M. Zhodzishsky and D. Milutin, 2010. The Equipment for High-Precision Positioning using Signals of Global Navigation Satellite Systems: Receivers and Consumers of Navigation Information. 1st Edn., MAI-Print, Mosow, ISBN-10: 978-5-7035-2233-2, pp: 292. 
Cohen, C., 1992. Attitude determination using GPS. Ph.D. Dissertation, Stanford University, Stanford.

Hodgart, M. and S. Purivigraipong, 2000. New approach to resolving instantaneous integer ambiguity resolution for spacecraft attitude determination using GPS signals. Proceedings of the Position Location and Navigation Symposium, Mar. 13-16, IEEE Xplore Press, San Diego, CA, pp: 132-139. DOI: 10.1109/PLANS.2000.838294

Kaplan, E. and C. Hegarty, 2006. Understanding GPS: Principles and Applications. 2nd Edn., Artech House, Boston, Mass, ISBN-10: 1580538940, pp: 703.

Knight, D., 1994. A new method of instantaneous ambiguity resolution. Proceedings of the 7 th International Technical Meeting of the Satellite Division of the Institute of Navigation, Sept. 20-23, Salt Lake City, UT, pp: 707-716.

Lin, D., L. Voon and N. Nagarajan, 2004. Real-time attitude determination for microsatellite by LAMBDA method combined with Kalman filtering. Proceedings of the 22nd AIAA International Communication Satellite Systems Conference, (SSC' 04), AIAA, pp: 8-8. DOI: 10.2514/6.2004-3118

Lipkin, I., 2008. Satellite Navigation Systems. 1st Edn., Vuzovskaya Kniga, Moscow, ISBN-10: 5-89522-147-5, pp: 288.

Misra, P. and P. Enge, 2012. Global Positioning System. Signals, Measurements and Performance. 2nd Edn., Ganaga-Jamuna Press, ISBN-10: 0-97095442-5, pp: 570 . n.a., 2011. IS-GPS-200F. Interface specification GPS space segment. User Segment.

Parkinson, B. and J. Spilker, 1996. Global positioning system: Theory and applications. AIAA.

Perov, A. and V. Kharisov, 2010. GLONASS: Principles of Construction and Operation. 1st Edn., Radiotekhnika, Moscow, ISBN-10: 978-5-88070-251-0, pp: 800.

Perov, A., 2012. Methods and Algorithms for Optimal Signal Reception in the Equipment of Satellite Navigation Systems Consumers. 1st Edn., Radiotekhnika, Moscow, ISBN-10: 978-5-88070-031-8, pp: 240.

Povalyaev, A., 2008. Satellite Radionavigation Systems: Time, Clock Readings, Formation of Measurements and Determination of the Coordinate. 1st Edn., Radiotekhnika, Moscow, ISBN-10: 978-5-88070-153-7, pp: 328.

Tikhonov, V. and V. Kharisov, 2004. Statistical Analysis and Synthesis of Radiotechnical Devices and Systems. 1st Edn., Radio and Communications, Moscow, ISBN-10: 5-256-00789-0, pp: 608.

Verhagen, S. and P. Teunissen, 2006. New global navigation satellite system ambiguity resolution method compared to existing approaches. Guidance Control Dynam., 29: 981-991. 